\title{
Autoimmune hepatitis in the elderly: Diagnosis and Pharmacological management
}

\author{
Syed Rizvi ${ }^{1}$ and Samer Gawrieh ${ }^{2}$
}

1. Division of Gastroenterology and Hepatology, Medical College of Wisconsin, Milwaukee, WI.

2. Division of Gastroenterology and Hepatology, Indiana University School of Medicine, Indianapolis, IN

Short Title: Autoimmune hepatitis in elderly

Word Count: $\quad$ (5028)

\author{
Corresponding Authors: \\ Syed Rizvi, MD \\ Gastroenterology \& Hepatology Division \\ Medical College of Wisconsin \\ 9200 West Wisconsin Avenue, \\ Milwaukee, WI 53226 \\ srizvi@mcw.edu \\ Samer Gawrieh, MD \\ Gastroenterology and Hepatology Division \\ Indiana University School of Medicine; \\ 702 Rotary Cir \\ Indianapolis, IN 46202-5175 \\ sgawrieh@iu.edu
}

This is the author's manuscript of the article published in final edited form as:

Rizvi, S., \& Gawrieh, S. (2018). Autoimmune Hepatitis in the Elderly: Diagnosis and Pharmacologic Management. Drugs \& Aging, 35(7), 589-602. https://doi.org/10.1007/s40266-018-0556-0 
Conflicts of Interests: Dr. Syed Rizvi and Dr. Samer Gawrieh declare no conflicts of interest relevant to this paper.

Source of Funding: Dr. Syed Rizvi and Dr. Samer Gawrieh received no external funding for this study.

Compliance with Ethics Guidelines: Dr. Syed Rizvi and Dr. Samer Gawrieh were compliant with the ethical standards in writing this paper. 


\section{ABSTRACT}

Autoimmune hepatitis (AIH) may present as acute or chronic hepatitis in the elderly. Advanced hepatic fibrosis and cirrhosis are common on first presentation in this population. In this review, we discuss the presentation, approach to diagnosis and management of $\mathrm{AlH}$ in the elderly. As poly-pharmacy is common in the elderly, careful medication use history is essential for detecting drug induced AlH-like hepatitis. Steroid sparing or minimizing therapeutic regimens are preferred to treat $\mathrm{AlH}$ in the elderly. For the purpose of induction, budesonide or lower dose prednisone in combination with AZA regimens are preferred over high dose prednisone monotherapy due to higher risks of side effects of the later in elderly. Goal of maintenance therapy should be to achieve a full biochemical and histologic remission. Bone density monitoring and interventions to prevent steroid-related bone disease should be implemented throughout the course of the disease. Liver transplantation should be considered in the elderly patient with liver failure or early hepatocellular carcinoma if there are no significant comorbidities or compromise in the functional status. 


\section{Key Points}

- AlH should be suspected in elderly patients presenting with chronically elevated LFTs

- Treatment is highly effective and results in survival benefit

- Azathioprine with budesonide or prenisone are commonly utilized treatment regimens for AlH

- Due to increased use of medications in elderly, drug induced autoimmune like hepatitis should be considered in the differential diagnosis of AlH

- Patients on treatment for AlH should be on Calcium and Vitamin D and early referral for Bisphosphonates use is recommended for those with osteoprosis

- Prednisone, budesonide and azathioprine are commonly used

- Elderly patients with liver failure or early liver cancer should be referred for liver transplantation evaluation if they are in good functional status and have no significant co-morbidities. 


\section{INTRODUCTION}

Autoimmune hepatitis $(\mathrm{AlH})$ is an autoreactive chronic inflammatory liver disease. Although it lacks a specific diagnostic marker, serologically it is associated with positive autoantibodies, primarily antinuclear antibody (ANA) and antismooth muscle antibody (ASMA) along with hypergammaglobulinemia. Histologically it is characterized by chronic hepatitis comprising mainly of plasma cell infiltrate. AlH has wide ranging presentations including acute elevation of liver enzymes, chronically elevated liver enzymes or in some cases "burnt out" cirrhosis which can affect the recognition of this disease. Although AlH is considered a disease of children and adolescents, about $20 \%$ of adults with AlH develop the disease after age $60 .{ }^{(1-3)}$ The male to female ratio is $1: 3$ in elderly patient with $\mathrm{AlH} .{ }^{(4)}$ The confidence in reaching the diagnosis is often influenced by the absence of specific lab tests which may cause delay in diagnosis. AlH-like hepatitis may also be triggered by certain toxins such as prescription medications and over the counter supplements. AlH must be considered in all patients of any age group with acute or chronically elevated transaminases. AlH is now recognized as a cause of graft dysfunction after orthotropic liver transplantation (OLTx). ${ }^{(5)}$

The definition of AlH in elderly has generally been described in most of the studies as AlH affecting patients $>60$ years of age. ${ }^{(7,15)}$ Challenges of managing $\mathrm{AlH}$ in the elderly starts with reaching the diagnosis and extend to the choice and side effects of pharmacological management in lieu of comorbid conditions, frequent presentation with cirrhosis, and potential drug interactions. ${ }^{(6)}$

This review addresses these challenges in managing $\mathrm{AlH}$ in the elderly and provides an outline of the diagnostic tools to reach an accurate diagnosis, indications for treating $\mathrm{AlH}$, updated treatment regimens to induce remission, and strategies to manage difficult to treat patients. We 
will also highlight $\mathrm{AlH}$-like hepatitis related to drugs used in elderly and also discuss the features of de novo AlH in elderly patients with prior history of liver transplantation for etiologies other than liver failure because of $\mathrm{AlH}$.

\section{CLINICAL PRESENTATION}

Elderly patients frequently present with more advanced degrees of hepatic fibrosis and cirrhosis. ${ }^{(1-}$

3) In a recent meta-analysis comparing 264 elderly patients with 592 younger patients with AlH, elderly patients were twice as likely to present without symptoms and $50 \%$ more likely to present with cirrhosis. ${ }^{(7)}$ Elderly patients may have acute or chronic presentations.

\subsection{Acute presentation}

Acute onset AlH may present as any other form of acute hepatitis with jaundice and other prodromal symptoms which could be confused with a viral illness. It could also present as fulminant liver failure. ${ }^{(8)}$ Acute onset AlH can be de novo acute inflammatory process or acute exacerbation of a chronic process..$^{(9,10)}$ Upon biopsy of the liver in patients presenting with acute onset of $\mathrm{AlH}$, panacinar hepatitis or centrilobular (zone 3) necrosis can be the only finding and may mislead the diagnosis. ${ }^{(11,12)}$ However, serial biopsies in such cases have shown transition into classic interface hepatitis. ${ }^{(13)}$ The reported incidence of acute $\mathrm{AlH}$ is $47-71 \%$ in elderly patients. ${ }^{(14)}$

\subsection{Chronic presentation:}

Elderly patients commonly present with advanced fibrosis and cirrhosis and may have signs of decompensated liver disease such as ascites. ${ }^{(1,2,7)}$ This suggests a more indolent form of chronic inflammation in these patients. Often this may be diagnosed as a part of work up for fatigue which is the commonest complaint found in $86 \%$ patients. ${ }^{(4)}$

Other nonspecific symptoms include upper abdominal discomfort in nearly $50 \%$ of patients and a 
third may present with anorexia and polymyalgia, while $25-34 \%$ patients may be asymptomatic on presentation. ${ }^{(15)}$

It should be noted that presence or absence of symptoms does not correlate with histologic grade of inflammation or stage of fibrosis. Hence, the decision to treat AlH should not rest on presence or absence of symptoms, but rather factor in biochemical, serologic and histologic markers of inflammation.

\subsection{Coexisting Diseases:}

Awareness of possible diseases that tend to occur in patients with AlH is important as they may influence the presentation, choice and potential side effects of therapy. Common coexisting diseases with AlH include celiac disease, autoimmune thyroiditis, Grave's disease, and rheumatoid arthritis. ${ }^{(16,17)}$

Elderly patients with celiac disease may not present with typical malabsorptive symptoms such as diarrhea, weight loss or abdominal pain. ${ }^{(18)}$ Micronutrient deficiency leading to osteoporosis may be the main complaint. ${ }^{(19,20)}$ Osteoporosis should be a strong reason to minimize or avoid systemic steroids in these AlH patients. AlH and other autoimmune cholestatic disorders are also more frequent in celiac disease than the general population. ${ }^{(21,22)}$ An entity called celiac hepatitis is a chronic rise in transaminases which may respond to gluten free diet. However, the concomitant AlH may require immunosuppressive treatment.

Concurrent thyroid disorders or rheumatic conditions are more often seen in elderly patients with $\mathrm{AlH}$ than in adults younger than 30 of age (42\%. versus $13 \%, \mathrm{p}=0.006) .{ }^{(13)}$ This may be explained by the presence of HLA-DRB1-04 which occurs more commonly in older white North American and Northern European patients. ${ }^{(2,15)}$ This particular HLA type is associated with more diverse antigenic peptides that predispose to concurrent autoimmune diseases found in elderly. ${ }^{(15}$, 
Primary Sclerosing Cholangitis (PSC) or Primary Biliary Cholangitis (PBC) may be coexisting in patients with autoimmune hepatitis. ${ }^{(24-26)}$ It should also be remembered that $5 \%$ of patients with AlH may have positive Antimitochondrial antibody (AMA) with absence of biliary changes defined as "serologic overlap" with no clinical implications. ${ }^{(27-32)}$ An unusually high alkaline phosphatase (ALP) should trigger investigation for an alternate diagnosis such as PBC or PSC as well as true overlap syndrome or mechanic biliary obstruction since only $21 \%$ of patients with true AlH have serum ALP more than twice the upper limit of normal and no patient with classic disease has serum ALP more than four times normal. ${ }^{(33)}$

\section{Drug induced liver injury presenting as AlH-like disease}

Drug induced liver injury (DILI) should be considered in the differential diagnosis of AlH. ${ }^{(34-39)}$ Differentiating drug induced $\mathrm{AlH}$-like hepatitis from classic $\mathrm{AlH}$ is often a challenge as unlike certain viral triggers for liver injury where specific serologic testing is available, diagnosis of this entity largely depends on clinical presentation and clinical judgement. AlH-like liver injury due to DILI may have one of the three following presentations: (1) A syndrome mimicking clinical, biochemical and serologic features of AlH which subsides after drug discontinuation; (2) Initiating autoimmune response to hepatobiliary antigens resulting in classic AlH; or (3) unmasking subclinical AlH. ${ }^{(34,35)}$

Although DILI may present at any age, the triggering medication may be of different class based on the age group. For example, Nitrofurantoin used for urinary tract infection in elderly females is a possible AlH trigger compared to Minocycline used for acne in younger females (Table.1) Furthermore, it is also well known that certain medications such as tumor necrosis factor-alpha inhibitors may unmask or exacerbate an already pre-existing $\mathrm{AlH}{ }^{(40,41)}$

TABLE 1: 
The elderly are at increased risk for drug toxicity due to increased frequency of chronic illnesses in this population which leads to utilization of multiple drugs. ${ }^{(42,43)}$ Cytochrome monooxygenase activity, particularly CYP3A levels decline in the elderly, ${ }^{(43)}$ also polypharmacy, ${ }^{(44)}$ renal insufficiency, ${ }^{(34)}$ and diminished hepatic blood flow ${ }^{(43)}$ are possible contributing factors to diminished drug metabolite clearance and increase drug toxicity in elderly. ${ }^{(35)}$

Clinicians should take an extensive history of prescription and over the counter medications used in past few months prior to liver enzymes elevation. Other clues which may point to DILI as a trigger for AlH include rash, fever and eosinophilia as seen in $20 \%$ to $30 \%$ of the cases. ${ }^{(45-}$ 48)

TABLE 2:

Drug induced $\mathrm{AlH}$ - like hepatitis usually resolves within 1 to 3 months after the offending medication is discontinued. ${ }^{(34,45,49,50)}$ This is in contrast to classic AlH where inflammation continues after drug withdrawal. ${ }^{(44)}$ In cases where corticosteroids were started due to severity of the disease and DILI remains a strong suspicion, the response of either drug induced autoimmune like hepatitis or classic AlH may be excellent, yet an important clue as drug being the cause of liver injury is the relapse of the disease after stopping steroids in cases of classic AlH. ${ }^{(51,52)}$ In some cases, however, particularly with biologic response modifiers (interferons, tumor necrosis factor antagonists), the AlH appears to have been triggered rather than caused 
by the drug and can be sustained unless long term immunosuppressive therapy is started. ${ }^{(53)}$

\section{DIAGNOSIS OF AIH}

\subsection{Serologic markers}

Antinuclear antibody (ANA) and Anti-smooth muscle antibody (ASMA) constitute 96\% of the antibodies present in patients with AlH. Anti-LKM antibody is often noted in European patients and is only present in a small proportion of the patients in North America. Absence of either antibody does not rule out the diagnosis of $\mathrm{AlH}$ in the correct setting. Patients who are seronegative may have positive antibodies at a later time. ${ }^{(54-58)}$ Also autoantibodies can be present with increasing propensity in otherwise healthy elderly individuals. ${ }^{(59-62)}$

Titers of autoantibodies have poor correlation with disease activity and treatment response. We do not recommend following the titers with treatment.

ANA and ASMA are the first antibodies with immunoglobulins $G$ which should be checked in patients with suspicion for AlH. In patients who test negative for these antibodies and suspicion for AlH remains high, checking anti-Soluble liver Antigen (Anti-SLA) ${ }^{(52,63-66)}$ and perinuclear anti neutrophil cytoplasmic antibody (pANCA) is done next. ${ }^{(67,68)}$ However, Anti-SLA antibody is more commonly found in association with conventional antibodies. ${ }^{(69-72)}$ If present Anti-SLA antibody points to more severe disease and worse outcomes.

\subsection{Diagnostic criteria of $\mathrm{AlH}$ :}

Two diagnostic criteria (Table. 3) are being validated and used to help with the diagnosis of AlH. The International Autoimmune Hepatitis Group (IAIHG) revised diagnostic criteria for AlH published in $1999^{(11)}$ and validated simplified diagnostic criteria published in $2008^{(44)}$ are 
complimentary to each other. In a large retrospective study, the specificities of the revised diagnostic criteria and simplified diagnostic criteria were observed to be the same $97.9 \%$ and 97\%, respectively. ${ }^{(73)}$ However, the former is considered more accurate in patients with atypical features of $\mathrm{AlH} .{ }^{(74,75)}$ Conversely, both are equally useful to diagnose AlH in patients with typical features of AlH.

TABLE 3

\section{TREATMENT}

The treatment indications and endpoints for $\mathrm{AlH}$ are the same as for young adults. The American Association for the Study of Liver Disease (AASLD) guidelines ${ }^{(67)}$ for treatment of AlH place the indications for treatment in 3 categories (Table.4):

5.1 Absolute indications for treatment: Three studies have shown that an AST level of at least $>10$ times upper limit of normal, or at least 5 times upper limit of normal with gamma globulin levels of more than two fold pose high mortality $(60 \%)$ at 6 months without treatment. Similarly, bridging necrosis or multilobular necrosis at presentation progress to cirrhosis in $82 \%$ of untreated patients with $45 \%$ mortality in 5 years. ${ }^{(76-78)}$ Incapacitating symptoms such as fatigue and arthralgia with hepatic inflammation are also absolute indications for treatment.

1. 5.2 Uncertain Indications: Patients with mild laboratory abnormality and histologic findings may have excellent immediate survival without treatment. ${ }^{(79,80)}$ Patients with inactive cirrhosis and are asymptomatic may also have favorable immediate survival without treatment. ${ }^{(81,82)}$ Similarly, asymptomatic patients without cirrhosis and evidence of inactive disease (mild elevation of transaminase) have 10 year survival exceeding $80 \% .{ }^{(81)}$ 
Use of treatment in these patients should be weighted based on comorbidities, degree of inflammatory activity and stage of fibrosis.

2. 5.3 No indications: Treatment is not indicated in patients with burnt out cirrhosis, in absence of inflammatory activity, and lack of symptoms, as the risk of drug induced side effects such as worsening of diabetes, vertebral compression fractures, severe cytopenia and osteoporosis is significantly elevated in elderly patients.

\section{TABLE 4:}

\subsection{Goals of treatment:}

The primary goal of therapy in AlH is to achieve remission. ${ }^{(67,83,84)}$ For practical purposes induction of remission is distinguished from maintenance of remission.

The AASLD practice guidelines ${ }^{(67)}$ define goals of remission as (a) normalization of transaminases, total bilirubin, gamma globulin and (b) absence of inflammatory activity on liver biopsy. Secondary endpoints include prevention of progression of fibrosis to cirrhosis and reversal of cirrhosis to a lower stage of fibrosis, an outcome which has been well documented with treatment of $\mathrm{AlH} .{ }^{(85,86)}$

\subsection{Treatment endpoints:}

There is no prescribed maximum or minimum duration of treatment. Conventional treatment is continued until remission, treatment failure, incomplete response or drug toxicity. ${ }^{(12,87)}$ 
Treatment goal should be to achieve biochemical and histologic remission. Nearly $90 \%$ of adults notice improvements in the serum AST, bilirubin and gamma-globin levels within 2 weeks. ${ }^{(88)}$ It should be noted that resolution of liver tests abnormalities and histologic inflammation rarely occurs in less than 1 year. The probability of remission diminishes after 2 year. ${ }^{\left({ }^{89-91)}\right.}$ It is also important to recognize that histologic improvement lags behind clinical and laboratory improvement by $3-8$ months. ${ }^{(92,93)}$ Therefore, hasty withdrawal of therapy is discouraged as it may result in relapse of $\mathrm{AlH}$. Cessation of therapy could be considered in patients who attained long term (18-24 months) remission, but confirmation of absent histological inflammatory activity prior to stopping therapy is essential to reduce the risk of relapse. ${ }^{(13,85,92,93)}$

Elderly patients aged $>60$ years are more likely to respond to steroid treatment than young adults (95\% vs $76 \%$ respectively; $p=0.03)$. Rate of remission ( $61 \%$ vs. $59 \%$ ) also occur with equal frequency in both groups. ${ }^{(2)}$

Despite reports of lower risk for AlH relapse with withdrawal of therapy after long remission, relapse could have detrimental effects on liver function and progression of liver disease in the elderly who frequently present with advanced fibrosis. To minimize these risks and make informed decisions about the risk of therapy cessation, we use liver biopsy to ensure histological remission before withdrawal of therapy. The overall health status of the elderly patients and complexity of comorbidities should be taken into account with these decisions.

\subsection{Drug related complications:}

Initial treatment regimens are well tolerated in elderly and post-menopausal women do not have statistically significant drug related side effects. $\left({ }^{92}, 93\right)$ However, use of steroids to treat the relapse in post-menopausal women is associated with higher cumulative frequency of drug related complications ( $77 \%$ vs. $48 \%$ respectively, $p<0.01)$. This includes vertebral compression (23\% vs. $7 \%, p=0.05)$ and lumbar spine densities below the spontaneous fracture threshold ( $85 \%$ vs. $22 \%, p=0.0002) .{ }^{(94)}$ In this regard it is worth emphasizing that although the individuals with osteoporosis are at the highest relative risk of fracture, majority of fractures occur in patients who have osteopenia and 
hence assessment of risk factors independent of bone mineral density be considered in these patients. Both men and women over age 50 are at risk for fractures due to these secondary risk factors which include chronic liver disease and hypogonadism, latter may happen in patients with advanced liver disease, prolonged steroid exposure and older age. (Lorraine A. FitzPatrick . Mayo Clin Proc, May 2002, Vol 77)

Additionally, this is worth emphasizing that risk of mortality with hip fractures (The components of excess mortality after hip fracture. Kanis JA, Oden A, Johnell O, De Laet C, Jonsson B, Oglesby AK . 2003;32(5):468); Meta-analysis: excess mortality after hip fracture among older women and men. Haentjens P, Magaziner J, Colón-Emeric CS, Vanderschueren D, Milisen K, Velkeniers B, Boonen S Ann Intern Med. 2010;152(6):380.

as well as vertebral fractures (Mortality after all major types of osteoporotic fracture in men and women: an observational study. Center JR, Nguyen TV, Schneider D, Sambrook PN, Eisman JA Lancet. 1999;353(9156):878. ) is much higher in men than in women. Hence, both genders are at risk of bone loss and subsequently fractures and be monitored closely.

Incidence rate of extrahepatic neoplasm in patients treated with AZA is 1 per 194 patient-years. The probability of tumor occurrence is $3 \%$ after 10 years which is 1.4 fold higher than that in age and sex matched controls. The risk of extrahepatic malignancy was not affected by age or sex. ${ }^{(95)}$

The most important AZA associated side effect is cytopenia which occurs with overall frequency of $46 \%$ however severe cases where treatment cessation is necessary occur in $6 \%$ of patients (NEW REFERENCE, ASK CATHY TO PUT IT CZAJA AJ, CARPERNTER HA. THIOPURINE METHYLTRANSFERASE DEFICIENCY AND AZATHIOPURINE INTOLERANCE IN AUTOIMMUNE HEPATITIS. DIG DIS SCI 2006;51:968-975. This risk is of significance in elderly patients because as mentioned previously incidence of cirrhosis at the time of diagnosis is higher in this group. Additionally in patients who developed relapse and required long term use of AZA to maintain remission, the risk of myelosuppression and lymphopenia is $7 \%$ and $57 \%$ 
respectively while the risk of malignancy of various cell types has been reported in $8 \%$ of patients. However, the association with the low dose of AZA used for long-term maintenance of remission in $\mathrm{AlH}$ remains uncertain. ${ }^{(96)}$

In a recent retrospective analysis on the use of mycophenolate mofetil (MMF) in patients with AlH 25\% developed adverse events, however serious adverse events occurred in only $2.7 \%$ patients, all of whom had cirrhosis ${ }^{\left({ }^{(84)}\right.}$ Caution should be used and close monitoring is advised when MMF is considered in patients with cirrhosis.

In a recent study evaluating the efficacy and safety of MMF and tacrolimus as second line therapy in patients with $\mathrm{AlH}, 12.5 \%$ (10/80) patients using tacrolimus (FK) developed side effects requiring therapy withdrawal. Most common was neurologic side effects in 4 patients followed by hypertension and generalized edema in 2 and gastrointestinal side effects in 2 patients. Renal failure was rare and noted in only one patient. ${ }^{(85)}$

A regular maintenance regimen to avoid bone disease should also be strongly considered in elderly patients receiving initial corticosteroid treatment. $(\underline{13,30,124)}$ This should include regular weight-bearing exercise, calcium (1-1.5 g/d), vitamin D3 (400-800 U/d), and consideration to add alendronate $(70 \mathrm{mg} / \mathrm{wk})$ as adjuvant therapy. Annual bone densitometry should be done in all elderly patients which may help decision on adjuvant bone regimens mentioned above. ${ }^{(15,97,}$ 98) 


\subsection{Is treatment in elderly of any benefit?}

The majority of patients (80-85\%) will respond to therapy. ${ }^{(99)}$ Although the rate of complete response, partial response and treatment failure is comparable to younger patients the relapse rate after treatment cessation is lower in the elderly than the younger counterparts (odds ratio $0.38) \cdot{ }^{(7)}$

Another advantage of therapy is the possibility in reduction of fibrosis along with controlling the inflammation. In one study on patients with mean age of 45 years, $56 \%$ patients followed for 55 +/- 9 months had improvement in fibrosis. ${ }^{(100)}$

These advantages of treatment favor initiation of treatment in elderly when indicated. Figure.1 shows the suggested treatment algorithm for treating $\mathrm{AlH}$ in the elderly.

Figure 1:

\subsection{Treatment regimens}

The 2010 AASLD guidelines recommend two regimens to be utilized for induction and maintenance of remission. (1) prednisone only; (2) prednisone in combination with AZA. Up to $44 \%$ of patients develop steroid related serious side effects with prednisone monotherapy. A lower number of patients $(\sim 10 \%)$ would still experience prednisone related serious side effects in AZA+ prednisone combination regimen. Hence, a third regimen containing budesonide in combination with AZA has been studied in comparison to the prednisone with AZA regimen and is now being recommended by several experts. ${ }^{(83,101)}$ In elderly patients our practice has been to use later two regimens to minimize or avoid steroids associated complications.

1. 5.8.1. Corticosteroid monotherapy: Prednisone alone can be started at the standard dose of $60 \mathrm{mg}$ daily. Once the liver enzymes start to improve, dose should 
be tapered down to an individual dose sufficient to maintain a remission from 20 $\mathrm{mg}$ daily onward, reduction should be done by $5 \mathrm{mg}$ every week until $10 \mathrm{mg} / \mathrm{day}$ is achieved. AZA can be added after week 4 to optimize immunosuppression. This strategy helps decrease the need for steroids and side effects related to steroids.

Situations where steroid monotherapy is the regimen of choice include patients with cytopenia (often seen in patients with cirrhosis), TPMT deficiency or malignancy. Due to increased prevalence of frailty, osteoporosis and higher predisposition to delirium and increased steroid toxicity in this population, this regimen is not the preferred first line option to treat $\mathrm{AlH}$ in the elderly in our practice. We also avoid the use of prednisone in doses higher than $30 \mathrm{mg}$ daily in patients with known underlying osteoporosis or poorly controlled diabetes.

5.8.2. Combination of prednisone and $A Z A$ : Another regimen to initiate treatment with is combination of prednisone $30 \mathrm{mg}$ daily with AZA $50 \mathrm{mg}$ daily, with prednisone is then be tapered by $5 \mathrm{mg}$ weekly after the end of week 2 (figure 1). A systemic review of randomized controlled trial showed equivalent results for induction of remission with prednisone alone or prednisone plus AZA regimen. ${ }^{(102)}$

5.8.3. Combination of budesonide and AZA: This strategy takes advantage of the first-pass metabolism of budesonide by the liver, ${ }^{(101,102)}$ which reduces the risk of systemic steroid toxicity. ${ }^{(103)}$ In a randomized, controlled double blinded multicenter phase $2 \mathrm{~b}$ trial of budesonide, ${ }^{(67)} 203$ non-cirrhotic patients, age 10-70 years with AlH received either AZA (1$2 \mathrm{mg} / \mathrm{kg} /$ day) plus either prednisone (40 $\mathrm{mg}$ a day tapering to $10 \mathrm{mg}$ a day or budesonide (9 mg a day) for 6 months. Patients who achieved complete biochemical response by 3 months and at the investigator's discretion, those not in remission by 6 months could proceed to part 
two of the study, a 6-month, open-label segment in which all patients received AZA and budesonide. At 6 months, more patients in the budesonide group than in the prednisone group achieved the primary endpoint: complete biochemical response (normalization of liver enzymes) $(60.0 \%$ vs. $38.8 \% ; P=0.001)$, and the absence of steroid-specific adverse effects, such as moon face, acne, buffalo hump, diabetes, and striae $(47.0 \%$ vs. $18.4 \% ; P<0.001)$. At 12 months, 95 (54.8\%) of 173 patients who completed part two of the study achieved complete response; rates of complete response were similar between patients originally randomized to budesonide or prednisone.

This regimen offers significant advantage in providing induction of remission compared to other regimen containing prednisone with $A Z A$, however at a much less rate of side effects. One major disadvantage of this regimen use is that it is unknown whether this regimen is effective in maintaining remission beyond 12 months because the primary efficacy endpoint was determined at 6 months. Also, budesonide is significantly more expensive than prednisone and may not be covered by all insurance plans.

Since its reported use 20 years ago, several small studies as well as case reports have been published regarding the use of budesonide. However, in these studies where budesonide was used as monotherapy, the clinical and biochemical remission rate was $9 \%$ to $58 \% .{ }^{(104-109)}$ Not all these studies reported steroids specific side effects consistently. However, an average of $30 \%$ of patients developed overall steroids related side effects. Another limitation of these studies is that the numbers were small and some used older definition of AASLD for biochemical remission (ALT $<2 x$ the upper limit of normal. Hence, due to the marginal efficacy if any with budesonide alone, it should not be used as monotherapy. ${ }^{(110)}$ Budesonide is contraindicated in patients with cirrhosis because portal systemic shunting and abnormal hepatic metabolism prevent complete hepatic first-pass extraction, reduce therapeutic efficacy, and cause systemic steroid side effects. ${ }^{(111)}$ Since elderly patients are more often 
diagnosed with cirrhosis than their younger counterparts; budesonide should not be used for induction with AZA unless cirrhosis is ruled out either with imaging or with liver biopsy. Also, budesonide should never be used in patients with cirrhosis for maintenance due to lack of evidence in long term effectiveness to maintain remission.

\subsection{Maintenance of remission}

Remission is maintained with either prednisone alone or AZA monotherapy or a combination of prednisone with AZA. One study showed combination of prednisone and AZA was superior in maintaining remission however the drug induced complications were higher in cirrhotic patients $(25 \%)$ than in non-cirrhotic patients (8\%). ${ }^{(67)}$ In another study AZA monotherapy was as effective as continuation of low dose steroids and AZA. ${ }^{(102)}$ Based on concern for long term use of steroids, higher probability of response in elderly we prefer AZA monotherapy to maintain remission.

Efficacy and safety of longer term use of budesonide and AZA longer than 12 months has not been studied in detail. However, to answer the efficacy of long term use of budesonide in patients with $\mathrm{AlH}$, Peiseler et al recently published their data on the largest cohort of $\mathrm{AlH}$ patients (60 patients) on long term (mean 31 months) budesonide. ${ }^{(112)}$ Budesonide was used as second line therapy in combination with either AZA or other immunosuppressive agents on patients who were either intolerant or dependent on prednisone. Long term remission was noted in only $40 \%$ of patients thereby doubling the initial remission rate. Bone density remained stable in majority of the patients. However, budesonide had to be switched back to prednisone in $23 \%$ of patients due to insufficient disease control or due to side effect in $13 \%$ of patients. One positive finding was that all 13 patients with full biochemical response on prednisone who were switched to budesonide either due to side effects or dependence on prednisone remained in biochemical remission. Since this was a retrospective study all the side effects related to budesonide were not 
recorded.

Every attempt should be made to taper the prednisone to the lowest dose possible in monotherapy regimen or to discontinue prednisone when using dual therapy with AZA after achieving the remission. Typically average time to reach the complete biochemical and histologic remission in elderly is 18 months. ${ }^{(90,94)}$ If this is not accomplished with standard dose AZA of $50 \mathrm{mg}$ a day we recommend titrating the dose of $A Z A$ to up to $2 \mathrm{mg} / \mathrm{kg} /$ day and monitoring the blood count while attempting to wean off the steroids. ${ }^{(96)}$

Hypoxanthine phoribosyltransferase leads to the production of 6 thioguanine nucleotide (6TGN) from 6MP which is formed as a result of non-enzymatic conversion of AZA. The 6TGN has immunosuppressive properties. TPMT and xanthine oxidase enzymatically convert 6-MP to 6thiouric acid and 6-methylmercaptopurine nucleotide (6-MMP), respectively. 6-TGN levels are indicators of patient adherence and useful for individualizing AZA doses. ${ }^{(113)}$ In a minority of nonresponders to AZA, 6-MP is preferentially metabolized by xanthine oxidase to nonimmunosuppressive 6-thiouric acid. Allopurinol which is an inhibitor of xanthine oxidase can redirect 6-MP metabolism to produce immunosuppressive 6-TGN. ${ }^{(114)}$ Hence, TPMT testing should be performed before labelling a patient a non-responder and considering the switch to an alternative regimen.

\subsection{Treatment failure and incomplete response}

Treatment failure is defined as worsening of AST or bilirubin, ongoing or worsening histologic activity on liver biopsy or new onset ascites and hepatic encephalopathy. ${ }^{(87)}$ 
Fortunately, the incidence of treatment failure is less common in elderly patients than their young counterparts ( $5 \%$ vs $24 \%$, respectively; $p=0.03){ }^{(2)}$

Incomplete response to therapy is defined as inability to achieve complete biochemical and histologic remission with conventional doses of prednisone or AZA.

Before resorting to alternative therapies, compliance with prescribed regimen should be assessed. Compliant patients who are non-responders to conventional immunosuppression could be treated with intensified and accelerated doses of conventional therapy. Checking thiopurine metabolite levels such as 6 TGN can prove useful in guiding therapy.

\subsection{Management of treatment failure}

One strategy to manage treatment failure is to increase the dose of prednisone to $60 \mathrm{mg}$ daily or increase AZA dose to up to $150 \mathrm{mg}$ daily in combination with prednisone $30 \mathrm{mg}$ daily for one month. ${ }^{(67)}$ Once the transaminases improve, prednisone dose should be decreased very gradually by $5 \mathrm{mg}$ weekly while monitoring the transaminases and Immunoglobulins to guide the pace of taper down to a dose of $10 \mathrm{mg}$ daily while monitoring the labs monthly. With this strategy, $70 \%$ patients experience biochemical and symptomatic improvement, although only $20 \%$ are able to achieve histologic remission. ${ }^{(67)}$

\subsection{Management of Incomplete response}

In such patients increasing the dose of AZA to up to $2 \mathrm{mg} / \mathrm{kg}$ daily should be attempted. Long term low dose corticosteroid therapy involves a gradual decrease in the prednisone dose by $2.5 \mathrm{mg}$ per month until the lowest level (ideally $5-10 \mathrm{mg}$ daily) is achieved or steroids can be safely discontinued and the serum ASA or ALT remain stable. ${ }^{(67)}$ 


\subsection{Alternate therapies for management of $\mathrm{AlH}$ in patients with intolerance, treatment failure or incomplete response to conventional therapies.}

Mycophenolate mofetil (MMF) is the prodrug of mycophenolic acid. Its efficacy in the management of AlH as an alternate therapy has been studied in smaller studies. Overall, patients who failed to respond to AZA were unlikely to achieve remission after switching to MMF. In contrast, MMF and mycophenolic acid appear to be effective for patients intolerant of $A Z A .{ }^{(115-118)}$ In a recent retrospective study of 105 patients who were switched to MMF, ${ }^{(119)} 60 \%$ achieved biochemical remission on MMF therapy with a median follow up period of 25 months. Response rates between patients who were switched because of inefficacy of standard therapy or treatment intolerance were similar ( $57 \%$ vs $62 \%$, respectively; $P=0.63$ ). Subjects with cirrhosis had a lower treatment response rate compared with those without cirrhosis, with biochemical remission achieved in $47 \%$ and $66 \%$ of subjects, respectively $(P=.07)$.

Another recent retrospective study of 201 patients followed in 19 centers who were either unable to tolerate the first line therapy (steroids or AZA) or had no response to conventional therapy were either treated with MMF or with tacrolimus (FK). ${ }^{(120)}$ Complete biochemical response was defined per AASLD guidelines. Overall response rate in patients who were unable to tolerate first line therapy showed similar $(69.4 \%$ vs $72.5 \%, p=0.63)$ complete biochemical remission to either MMF or FK. However, significantly more non-responders to conventional therapy achieved biochemical remission with FK compared to MMF $(56.5 \%$ vs $34 \%$, respectively; $P=0.029)$. The rates of complete response were significantly lower in non-responders than in patients intolerant to conventional therapy, both for patients treated with MMF and for patients treated with FK $(\mathrm{P}<$ .001). Hence for patients who have failed conventional therapies consideration should be given to switch to MMF or FK as second line therapy. Treatment with FK may achieve higher rate of biochemical remission in those with suboptimal response to conventional therapy compared to 
MMF.

\subsection{ORTHOTROPIC LIVER TRANSPLANTATION IN ELDERLY:}

OLTx for AlH provides excellent 5-year and 10 -year survivals of $>70 \%$ for adults with AlH. ${ }^{(121-123)}$ In a European study, 5 year survival after OLTx for AlH in 827 patients was $73 \%$. Interestingly five-year survival after OLT for AlH varied inversely with age, 78\% (95\% confidence interval, $70 \%-86 \%)$ for ages $18-34$ vs $61 \%$ (95\% confidence interval, $51 \%-70 \%)$ for age $>50$ years. This is likely due to the higher rate of infections after transplant in older patients transplanted with AlH $(\mathrm{HR} 1.8 ; \mathrm{P}=.002){ }^{(122)}$

Only $6 \%$ of liver transplants performed in the US are done in patients with $\mathrm{AlH}^{(124)}$ When an elderly patient is considered for liver transplantation, the patient's functional status and other comorbidities should be thoroughly considered in the decision to determine candidacy for transplant. We emphasize overall health and functional status as indicators of patient's physiological age and place less emphasis on patient's chronological age in determining candidacy for liver transplant.

\section{CONCLUSION}

AlH should be considered in patients older than 60 who present with acute or chronic hepatitis. Elderly patients with $\mathrm{AlH}$ frequently present with advanced hepatic fibrosis and cirrhosis. Diagnosis of AlH can be made with accuracy by using one of the two diagnostic criteria. Treatment of AlH in elderly should be based on the strict criteria set by AASLD guidelines. High doses of prednisone are avoided when possible. Preferred treatment options in the elderly are the combinations of prednisone with AZA or budesonide with AZA. These regimens require less 
cumulative dose of steroids than prednisone monotherapy. Elderly patients with liver failure or early hepatocellular carcinoma should be considered for liver transplantation if in good functional status and have no significant comorbidities.

\section{References:}

1. Al-Chalabi T, Boccato S, Portmann BC, McFarlane IG, Heneghan MA. Autoimmune hepatitis (AIH) in the elderly: a systematic retrospective analysis of a large group of consecutive patients with definite AlH followed at a tertiary referral centre. J Hepatol. 2006;45(4):575-83.

2. Czaja AJ, Carpenter HA. Distinctive clinical phenotype and treatment outcome of type 1 autoimmune hepatitis in the elderly. Hepatology. 2006;43(3):532-8.

3. Schramm C, Kanzler S, zum Buschenfelde KH, Galle PR, Lohse AW. Autoimmune hepatitis in the elderly. Am J Gastroenterol. 2001;96(5):1587-91.

4. Verslype C, George C, Buchel E, Nevens F, van Steenbergen W, Fevery J. Diagnosis and treatment of autoimmune hepatitis at age 65 and older. Aliment Pharmacol Ther. 2005;21(6):695-9.

5. Czaja AJ. Diverse manifestations and evolving treatments of autoimmune hepatitis. Minerva Gastroenterol Dietol. 2005;51(4):313-33.

6. Czaja AJ. Progress in the diagnosis and treatment of autoimmune hepatitis. Minerva Med. 2008;99(6):549-68.

7. Chen J, Eslick GD, Weltman M. Systematic review with meta-analysis: clinical manifestations and management of autoimmune hepatitis in the elderly. Aliment Pharmacol Ther. 2014;39(2):117-24.

8. Kessler WR, Cummings OW, Eckert G, Chalasani N, Lumeng L, Kwo PY. Fulminant hepatic failure as the initial presentation of acute autoimmune hepatitis. Clin Gastroenterol Hepatol. 2004;2(7):625-31.

9. Burgart L, Batts KP, Ludwig J, Nikias GA, Czaja AJ. Recent-onset autoimmune hepatitis. Biopsy findings and clinical correlations. Am J Surg Pathol. 1995;19(6):699-708.

10. Nikias GA, Batts KP, Czaja AJ. The nature and prognostic implications of autoimmune hepatitis with an acute presentation. J Hepatol. 1994;21(5):866-71.

11. Alvarez F, Berg PA, Bianchi FB, Bianchi L, Burroughs AK, Cancado EL, et al. International Autoimmune Hepatitis Group Report: review of criteria for diagnosis of autoimmune hepatitis. J Hepatol. 1999;31(5):929-38.

12. Czaja AJ, Freese DK, American Association for the Study of Liver D. Diagnosis and treatment of autoimmune hepatitis. Hepatology. 2002;36(2):479-97. 
13. Okano N, Yamamoto K, Sakaguchi K, Miyake Y, Shimada N, Hakoda T, et al. Clinicopathological features of acute-onset autoimmune hepatitis. Hepatol Res. 2003;25(3):263-70.

14. Parker DR, Kingham JG. Type I autoimmune hepatitis is primarily a disease of later life. QJM. 1997;90(4):289-96.

15. Czaja AJ. Clinical features, differential diagnosis and treatment of autoimmune hepatitis in the elderly. Drugs Aging. 2008;25(3):219-39.

16. Villalta D, Girolami D, Bidoli E, Bizzaro N, Tampoia M, Liguori M, et al. High prevalence of celiac disease in autoimmune hepatitis detected by anti-tissue tranglutaminase autoantibodies. J Clin Lab Anal. 2005;19(1):6-10.

17. Volta U, De Franceschi L, Molinaro N, Cassani F, Muratori L, Lenzi M, et al. Frequency and significance of anti-gliadin and anti-endomysial antibodies in autoimmune hepatitis. Dig Dis Sci. 1998;43(10):2190-5.

18. Cranney A, Zarkadas M, Graham ID, Butzner JD, Rashid M, Warren R, et al. The Canadian Celiac Health Survey. Dig Dis Sci. 2007;52(4):1087-95.

19. Bullamore JR, Wilkinson R, Gallagher JC, Nordin BE, Marshall DH. Effect of age on calcium absorption. Lancet. 1970;2(7672):535-7.

20. Gallagher JC, Riggs BL, Eisman J, Hamstra A, Arnaud SB, DeLuca HF. Intestinal calcium absorption and serum vitamin $D$ metabolites in normal subjects and osteoporotic patients: effect of age and dietary calcium. J Clin Invest. 1979;64(3):729-36.

21. Rubio-Tapia A, Murray JA. Liver involvement in celiac disease. Minerva Med. 2008;99(6):595-604.

22. Volta U, Rodrigo L, Granito A, Petrolini N, Muratori P, Muratori L, et al. Celiac disease in autoimmune cholestatic liver disorders. Am J Gastroenterol. 2002;97(10):2609-13.

23. Al-Chalabi T, Underhill JA, Portmann BC, McFarlane IG, Heneghan MA. Impact of gender on the long-term outcome and survival of patients with autoimmune hepatitis. J Hepatol. 2008;48(1):140-7.

24. Kaya $M$, Angulo $P$, Lindor KD. Overlap of autoimmune hepatitis and primary sclerosing cholangitis: an evaluation of a modified scoring system. J Hepatol. 2000;33(4):537-42.

25. Omagari K, Masuda J, Kato Y, Nakata K, Kanematsu T, Kusumoto Y, et al. Re-analysis of clinical features of 89 patients with autoimmune hepatitis using the revised scoring system proposed by the International Autoimmune Hepatitis Group. Intern Med. 2000;39(12):1008-12.

26. van Buuren HR, van Hoogstraten HJE, Terkivatan T, Schalm SW, Vleggaar FP. High prevalence of autoimmune hepatitis among patients with primary sclerosing cholangitis. J Hepatol. 2000;33(4):543-8.

27. Czaja AJ, Carpenter HA, Manns MP. Antibodies to soluble liver antigen, P450IID6, and mitochondrial complexes in chronic hepatitis. Gastroenterology. 1993;105(5):1522-8.

28. Kenny RP, Czaja AJ, Ludwig J, Dickson ER. Frequency and significance of antimitochondrial antibodies in severe chronic active hepatitis. Dig Dis Sci. 1986;31(7):705-11.

29. Mishima S, Omagari K, Ohba K, Kadokawa Y, Masuda J, Mishima R, et al. Clinical implications of antimitochondrial antibodies in type 1 autoimmune hepatitis: a longitudinal study. Hepatogastroenterology. 2008;55(81):221-7.

30. Montano-Loza AJ, Carpenter HA, Czaja AJ. Frequency, behavior, and prognostic implications of antimitochondrial antibodies in type 1 autoimmune hepatitis. J Clin Gastroenterol. 2008;42(9):1047-53.

31. Nezu S, Tanaka A, Yasui H, Imamura M, Nakajima $\mathrm{H}$, Ishida $\mathrm{H}$, et al. Presence of antimitochondrial autoantibodies in patients with autoimmune hepatitis. J Gastroenterol Hepatol. 2006;21(9):1448-54.

32. O'Brien C, Joshi S, Feld JJ, Guindi M, Dienes HP, Heathcote EJ. Long-term follow-up of antimitochondrial antibody-positive autoimmune hepatitis. Hepatology. 2008;48(2):550-6.

33. Czaja AJ. Frequency and nature of the variant syndromes of autoimmune liver disease. Hepatology. 1998;28(2):360-5.

34. Bjornsson E, Talwalkar J, Treeprasertsuk S, Kamath PS, Takahashi N, Sanderson S, et al. Druginduced autoimmune hepatitis: clinical characteristics and prognosis. Hepatology. 2010;51(6):2040-8. 
35. Czaja AJ. Drug-induced autoimmune-like hepatitis. Dig Dis Sci. 2011;56(4):958-76.

36. Fujiwara K, Yokosuka O. Histological discrimination between autoimmune hepatitis and druginduced liver injury. Hepatology. 2012;55(2):657.

37. Kaymakoglu S. Drug-induced hepatitis, drug-induced autoimmunity or classical autoimmune hepatitis: how can we differentiate? Turk J Gastroenterol. 2004;15(3):123-5.

38. Lucena MI, Kaplowitz N, Hallal H, Castiella A, Garcia-Bengoechea M, Otazua P, et al. Recurrent drug-induced liver injury (DILI) with different drugs in the Spanish Registry: the dilemma of the relationship to autoimmune hepatitis. J Hepatol. 2011;55(4):820-7.

39. Suzuki A, Brunt EM, Kleiner DE, Miquel R, Smyrk TC, Andrade RJ, et al. The use of liver biopsy evaluation in discrimination of idiopathic autoimmune hepatitis versus drug-induced liver injury. Hepatology. 2011;54(3):931-9.

40. Sokolove J, Strand V, Greenberg JD, Curtis JR, Kavanaugh A, Kremer JM, et al. Risk of elevated liver enzymes associated with TNF inhibitor utilisation in patients with rheumatoid arthritis. Ann Rheum Dis. 2010;69(9):1612-7.

41. Mancini S, Amorotti E, Vecchio S, de Leon MP, Roncucci L. Infliximab-related hepatitis: discussion of a case and review of the literature. Intern Emerg Med. 2010;5(3):193-200.

42. Chalasani N, Bjornsson E. Risk factors for idiosyncratic drug-induced liver injury. Gastroenterology. 2010;138(7):2246-59.

43. Cotreau MM, von Moltke LL, Greenblatt DJ. The influence of age and sex on the clearance of cytochrome P450 3A substrates. Clin Pharmacokinet. 2005;44(1):33-60.

44. Hennes EM, Zeniya M, Czaja AJ, Pares A, Dalekos GN, Krawitt EL, et al. Simplified criteria for the diagnosis of autoimmune hepatitis. Hepatology. 2008;48(1):169-76.

45. Lewis JH, Zimmerman HJ. Drug-induced autoimmune liver disease. In: Krawitt EL, Wiesner R, Nishioka K, editors. Autoimmune Liver Diseases. 2nd ed. Amsterdam: Elsevier; 1998. p. 627-49.

46. Liu ZX, Kaplowitz N. Immune-mediated drug-induced liver disease. Clin Liver Dis. 2002;6(3):755-

74.

47. Andrade RJ, Lucena MI, Fernandez MC, Pelaez G, Pachkoria K, Garcia-Ruiz E, et al. Drug-induced liver injury: an analysis of 461 incidences submitted to the Spanish registry over a 10-year period. Gastroenterology. 2005;129(2):512-21.

48. Watkins PB, Seeff LB. Drug-induced liver injury: summary of a single topic clinical research conference. Hepatology. 2006;43(3):618-31.

49. Bjornsson E, Davidsdottir L. The long-term follow-up after idiosyncratic drug-induced liver injury with jaundice. J Hepatol. 2009;50(3):511-7.

50. Bjornsson E, Kalaitzakis E, Av Klinteberg V, Alem N, Olsson R. Long-term follow-up of patients with mild to moderate drug-induced liver injury. Aliment Pharmacol Ther. 2007;26(1):79-85.

51. Hegarty JE, Nouri Aria KT, Portmann B, Eddleston AL, Williams R. Relapse following treatment withdrawal in patients with autoimmune chronic active hepatitis. Hepatology. 1983;3(5):685-9.

52. Zauli D, Ghetti S, Grassi A, Descovich C, Cassani F, Ballardini G, et al. Anti-neutrophil cytoplasmic antibodies in type 1 and 2 autoimmune hepatitis. Hepatology. 1997;25(5):1105-7.

53. [Available from: https://livertox.nih.gov/Phenotypes auto.html.

54. Czaja AJ, Carpenter HA, Santrach PJ, Moore SB, Homburger HA. The nature and prognosis of severe cryptogenic chronic active hepatitis. Gastroenterology. 1993;104(6):1755-61.

55. Czaja AJ, Rakela J, Hay JE, Moore SB. Clinical and prognostic implications of HLA B8 in corticosteroid-treated severe autoimmune chronic active hepatitis. Gastroenterology. 1990;98(6):158793.

56. Gassert DJ, Garcia H, Tanaka K, Reinus JF. Corticosteroid-responsive cryptogenic chronic hepatitis: evidence for seronegative autoimmune hepatitis. Dig Dis Sci. 2007;52(9):2433-7. 
57. Potthoff A, Deterding K, Trautwein C, Flemming P, Strassburg CP, Manns MP, et al. Steroid treatment for severe acute cryptogenic hepatitis. Z Gastroenterol. 2007;45(1):15-9.

58. Provenzano G, Marino L, Craxi A. Features of autoimmunity in cryptogenic chronic hepatitis. Allergol Immunopathol (Madr). 1991;19(3):119-22.

59. Rowley MJ, Buchanan H, Mackay IR. Reciprocal change with age in antibody to extrinsic and intrinsic antigens. Lancet. 1968;2(7558):24-6.

60. Hooper B, Whittingham S, Mathews JD, Mackay IR, Curnow DH. Autoimmunity in a rural community. Clin Exp Immunol. 1972;12(1):79-87.

61. Talor E, Rose NR. Hypothesis: the aging paradox and autoimmune disease. Autoimmunity. 1991;8(3):245-9.

62. Currie MS. Immunosenescence. Compr Ther. 1992;18(11):26-34.

63. Duerr RH, Targan SR, Landers CJ, LaRusso NF, Lindsay KL, Wiesner RH, et al. Neutrophil cytoplasmic antibodies: a link between primary sclerosing cholangitis and ulcerative colitis. Gastroenterology. 1991;100(5 Pt 1):1385-91.

64. Frenzer A, Fierz W, Rundler E, Hammer B, Binek J. Atypical, cytoplasmic and perinuclear antineutrophil cytoplasmic antibodies in patients with inflammatory bowel disease. J Gastroenterol Hepatol. 1998;13(9):950-4.

65. LaBrecque D, Phillips M, Ippolito L, Mitros F, Goeken J. Antineutrophil cytoplasmic antibody and chronic liver disease. Hepatology. 1999;30:428A.

66. Targan SR, Landers C, Vidrich A, Czaja AJ. High-titer antineutrophil cytoplasmic antibodies in type1 autoimmune hepatitis. Gastroenterology. 1995;108(4):1159-66.

67. Manns MP, Czaja AJ, Gorham JD, Krawitt EL, Mieli-Vergani G, Vergani D, et al. Diagnosis and management of autoimmune hepatitis. Hepatology. 2010;51(6):2193-213.

68. Wies I, Brunner S, Henninger J, Herkel J, Kanzler S, Meyer zum Buschenfelde KH, et al. Identification of target antigen for SLA/LP autoantibodies in autoimmune hepatitis. Lancet. 2000;355(9214):1510-5.

69. Baeres M, Herkel J, Czaja AJ, Wies I, Kanzler S, Cancado EL, et al. Establishment of standardised SLA/LP immunoassays: specificity for autoimmune hepatitis, worldwide occurrence, and clinical characteristics. Gut. 2002;51(2):259-64.

70. Ma Y, Bogdanos BP, Wiliams R, Mieli-Vergani G, Vergani D. Anti-SLA antibody is a marker of severity of liver damage in patients with autoimmune liver disease. J Hepatol. 2001;34(Suppl 1).

71. Torres-Collado AX, Czaja AJ, Gelpi C. Anti-tRNP(ser)sec/SLA/LP autoantibodies. Comparative study using in-house ELISA with a recombinant $48.8 \mathrm{kDa}$ protein, immunoblot, and analysis of immunoprecipitated RNAs. Liver Int. 2005;25(2):410-9.

72. Vitozzi S, Djilali-Saiah I, Lapierre P, Alvarez F. Anti-soluble liver antigen/liver-pancreas (SLA/LP) antibodies in pediatric patients with autoimmune hepatitis. Autoimmunity. 2002;35(8):485-92.

73. Gatselis NK, Zachou K, Papamichalis P, Koukoulis GK, Gabeta S, Dalekos GN, et al. Comparison of simplified score with the revised original score for the diagnosis of autoimmune hepatitis: a new or a complementary diagnostic score? Dig Liver Dis. 2010;42(11):807-12.

74. Czaja AJ. Comparability of probable and definite autoimmune hepatitis by international diagnostic scoring criteria. Gastroenterology. 2011;140(5):1472-80.

75. Longhi MS, Ma Y, Mieli-Vergani G, Vergani D. Aetiopathogenesis of autoimmune hepatitis. J Autoimmun. 2010;34(1):7-14.

76. Baggenstoss AH, Soloway RD, Summerskill WH, Elveback LR, Schoenfield L. Chronic active liver disease. The range of histologic lesions, their response to treatment, and evolution. Hum Pathol. 1972;3(2):183-98.

77. Cooksley WG, Bradbear RA, Robinson W, Harrison M, Halliday JW, Powell LW, et al. The prognosis of chronic active hepatitis without cirrhosis in relation to bridging necrosis. Hepatology. 1986;6(3):345-8. 
78. Schalm SW, Korman MG, Summerskill WH, Czaja AJ, Baggenstoss AH. Severe chronic active liver disease. Prognostic significance of initial morphologic patterns. Am J Dig Dis. 1977;22(11):973-80.

79. Czaja AJ. Features and consequences of untreated type 1 autoimmune hepatitis. Liver Int. 2009;29(6):816-23.

80. Koretz RL, Lewin KJ, Higgins J, Fagen ND, Gitnick GL. Chronic active hepatitis. Who meets treatment criteria? Dig Dis Sci. 1980;25(9):695-9.

81. Feld JJ, Dinh H, Arenovich T, Marcus VA, Wanless IR, Heathcote EJ. Autoimmune hepatitis: effect of symptoms and cirrhosis on natural history and outcome. Hepatology. 2005;42(1):53-62.

82. Kogan J, Safadi R, Ashur Y, Shouval D, Ilan Y. Prognosis of symptomatic versus asymptomatic autoimmune hepatitis: a study of 68 patients. J Clin Gastroenterol. 2002;35(1):75-81.

83. Manns MP, Lohse AW, Vergani D. Autoimmune hepatitis--Update 2015. J Hepatol. 2015;62(1 Suppl):S100-11.

84. Sahebjam F, Vierling JM. Autoimmune hepatitis. Front Med. 2015;9(2):187-219.

85. Czaja AJ. Review article: The prevention and reversal of hepatic fibrosis in autoimmune hepatitis. Aliment Pharmacol Ther. 2014;39(4):385-406.

86. Shah AM, Malhotra A, Kothari S, Baddoura W, Depasquale J, Spira R. Reversal of liver cirrhosis in autoimmune hepatitis. Hepatogastroenterology. 2011;58(112):2115-7.

87. Czaja AJ. Treatment strategies in autoimmune hepatitis. Clin Liver Dis. 2002;6(3):799-824.

88. Czaja AJ, Rakela J, Ludwig J. Features reflective of early prognosis in corticosteroid-treated severe autoimmune chronic active hepatitis. Gastroenterology. 1988;95(2):448-53.

89. Czaja AJ, Ammon HV, Summerskill WH. Clinical features and prognosis of severe chronic active liver disease (CALD) after corticosteroid-induced remission. Gastroenterology. 1980;78(3):518-23.

90. Czaja AJ, Beaver SJ, Shiels MT. Sustained remission after corticosteroid therapy of severe hepatitis B surface antigen-negative chronic active hepatitis. Gastroenterology. 1987;92(1):215-9.

91. Czaja AJ, Menon KV, Carpenter HA. Sustained remission after corticosteroid therapy for type 1 autoimmune hepatitis: a retrospective analysis. Hepatology. 2002;35(4):890-7.

92. Czaja AJ, Wolf AM, Baggenstoss AH. Laboratory assessment of severe chronic active liver disease during and after corticosteroid therapy: correlation of serum transaminase and gamma globulin levels with histologic features. Gastroenterology. 1981;80(4):687-92.

93. Soloway RD, Summerskill WH, Baggenstoss AH, Geall MG, Gitnick GL, Elveback IR, et al. Clinical, biochemical, and histological remission of severe chronic active liver disease: a controlled study of treatments and early prognosis. Gastroenterology. 1972;63(5):820-33.

94. Wang KK, Czaja AJ. Prognosis of corticosteroid-treated hepatitis B surface antigen-negative chronic active hepatitis in postmenopausal women: a retrospective analysis. Gastroenterology. 1989;97(5):1288-93.

95. Wang KK, Czaja AJ, Beaver SJ, Go VL. Extrahepatic malignancy following long-term immunosuppressive therapy of severe hepatitis B surface antigen-negative chronic active hepatitis. Hepatology. 1989;10(1):39-43.

96. Johnson PJ, McFarlane IG, Williams R. AZA for long-term maintenance of remission in autoimmune hepatitis. N Engl J Med. 1995;333(15):958-63.

97. Czaja AJ. Safety issues in the management of autoimmune hepatitis. Expert Opin Drug Saf. 2008;7(3):319-33.

98. Czaja AJ. Special clinical challenges in autoimmune hepatitis: the elderly, males, pregnancy, mild disease, fulminant onset, and nonwhite patients. Semin Liver Dis. 2009;29(3):315-30.

99. Yeoman AD, Longhi MS, Heneghan MA. Review article: the modern management of autoimmune hepatitis. Aliment Pharmacol Ther. 2010;31(8):771-87.

100. Czaja AJ, Carpenter HA. Decreased fibrosis during corticosteroid therapy of autoimmune hepatitis. J Hepatol. 2004;40(4):646-52. 
101. Vierling JM. Autoimmune Hepatitis and Overlap Syndromes: Diagnosis and Management. Clin Gastroenterol Hepatol. 2015;13(12):2088-108.

102. Lamers MM, van Oijen MG, Pronk M, Drenth JP. Treatment options for autoimmune hepatitis: a systematic review of randomized controlled trials. J Hepatol. 2010;53(1):191-8.

103. Strassburg CP. Therapeutic options to treat autoimmune hepatitis in 2009. Dig Dis. 2010;28(1):938.

104. Csepregi A, Rocken C, Treiber G, Malfertheiner P. Budesonide induces complete remission in autoimmune hepatitis. World J Gastroenterol. 2006;12(9):1362-6.

105. Czaja AJ, Lindor KD. Failure of budesonide in a pilot study of treatment-dependent autoimmune hepatitis. Gastroenterology. 2000;119(5):1312-6.

106. Danielsson A, Prytz $\mathrm{H}$. Oral budesonide for treatment of autoimmune chronic active hepatitis. Aliment Pharmacol Ther. 1994;8(6):585-90.

107. Delgado JS, Vodonos A, Malnick S, Kriger O, Wilkof-Segev R, Delgado B, et al. Autoimmune hepatitis in southern Israel: a 15-year multicenter study. J Dig Dis. 2013;14(11):611-8.

108. Peiseler M, Liebscher T, Sebode M, Zenouzi R, Hartl J, Ehlken H, et al. Efficacy and Limitations of Budesonide as a Second-Line Treatment for Patients With Autoimmune Hepatitis. Clin Gastroenterol Hepatol. 2018;16(2):260-7 e1.

109. Wiegand J, Schuler A, Kanzler S, Lohse A, Beuers U, Kreisel W, et al. Budesonide in previously untreated autoimmune hepatitis. Liver Int. 2005;25(5):927-34.

110. Lohse AW, Gil H. Reactivation of autoimmune hepatitis during budesonide monotherapy, and response to standard treatment. J Hepatol. 2011;54(4):837-9.

111. Geier A, Gartung C, Dietrich CG, Wasmuth HE, Reinartz P, Matern S. Side effects of budesonide in liver cirrhosis due to chronic autoimmune hepatitis: influence of hepatic metabolism versus portosystemic shunts on a patient complicated with HCC. World J Gastroenterol. 2003;9(12):2681-5.

112. Peiseler M, Liebscher T, Sebode M, Zenouzi R, Hartl J, Ehlken H, et al. Efficacy and Limitations of Budesonide as a Second-Line Treatment for Patients With Autoimmune Hepatitis. Clin Gastroenterol Hepatol. 2017.

113. Hindorf $\mathrm{U}$, Jahed K, Bergquist A, Verbaan H, Prytz $\mathrm{H}$, Wallerstedt $\mathrm{S}$, et al. Characterisation and utility of thiopurine methyltransferase and thiopurine metabolite measurements in autoimmune hepatitis. J Hepatol. 2010;52(1):106-11.

114. de Boer YS, van Gerven NM, de Boer NK, Mulder CJ, Bouma G, van Nieuwkerk CM. Allopurinol safely and effectively optimises thiopurine metabolites in patients with autoimmune hepatitis. Aliment Pharmacol Ther. 2013;37(6):640-6.

115. Baven-Pronk AM, Coenraad MJ, van Buuren HR, de Man RA, van Erpecum KJ, Lamers MM, et al. The role of mycophenolate mofetil in the management of autoimmune hepatitis and overlap syndromes. Aliment Pharmacol Ther. 2011;34(3):335-43.

116. Chatur N, Ramji A, Bain VG, Ma MM, Marotta PJ, Ghent CN, et al. Transplant immunosuppressive agents in non-transplant chronic autoimmune hepatitis: the Canadian association for the study of liver (CASL) experience with mycophenolate mofetil and tacrolimus. Liver Int. 2005;25(4):723-7.

117. Inductivo-Yu I, Adams A, Gish RG, Wakil A, Bzowej NH, Frederick RT, et al. Mycophenolate mofetil in autoimmune hepatitis patients not responsive or intolerant to standard immunosuppressive therapy. Clin Gastroenterol Hepatol. 2007;5(7):799-802.

118. Sharzehi K, Huang MA, Schreibman IR, Brown KA. Mycophenolate mofetil for the treatment of autoimmune hepatitis in patients refractory or intolerant to conventional therapy. Can J Gastroenterol. 2010;24(10):588-92.

119. Roberts SK, Lim R, Strasser S, Nicoll A, Gazzola A, Mitchell J, et al. Efficacy and Safety of Mycophenolate Mofetil in Patients With Autoimmune Hepatitis and Suboptimal Outcomes After Standard Therapy. Clin Gastroenterol Hepatol. 2017. 
120. Efe C, Hagstrom H, Ytting H, Bhanji R, Muller N, Wang Q, et al. Efficacy and Safety of Mycophenolate Mofetil and Tacrolimus as Second-line Therapy for Patients With Autoimmune Hepatitis. Clin Gastroenterol Hepatol. 2017;15(12):1950-6.

121. Ilyas JA, O'Mahony CA, Vierling JM. Liver transplantation in autoimmune liver diseases. Best Pract Res Clin Gastroenterol. 2011;25(6):765-82.

122. Schramm C, Bubenheim M, Adam R, Karam V, Buckels J, O'Grady JG, et al. Primary liver transplantation for autoimmune hepatitis: a comparative analysis of the European Liver Transplant Registry. Liver Transpl. 2010;16(4):461-9.

123. Tanaka T, Sugawara Y, Kokudo N. Liver transplantation and autoimmune hepatitis. Intractable Rare Dis Res. 2015;4(1):33-8.

124. Seaberg EC, Belle SH, Beringer KC, Schivins JL, Detre KM. Liver transplantation in the United States from 1987-1998: updated results from the Pitt-UNOS Liver Transplant Registry. Clin Transpl. 1998:17-37. 